\title{
Static Modulation Wave of Parallel Self-Assembled Arrays Transduced to a Hierarchy of Nanoscale Change Stimuli of Crystalline Rotors Dynamics
}

\author{
Sergey Simonov, ${ }^{1,2}$ Leokadiya Zorina, ${ }^{1,2}$ Pawel Wzietek, ${ }^{* 3}$ Antonio Rodríguez-Fortea, ${ }^{4}$ Enric \\ Canadell, ${ }^{* 5}$ Cécile Mézière, ${ }^{1}$ Guillaume Bastien, ${ }^{1}$ Cyprien Lemouchi, ${ }^{1}$ Miguel A. Garcia- \\ Garibay, ${ }^{* 6}$ and Patrick Batail ${ }^{* 1}$
}

\begin{abstract}
Affiliations:
${ }^{1}$ Laboratoire MOLTECH-Anjou, CNRS UMR 6200, Université d'Angers, France ${ }^{2}$ Institute of Solid State Physics, Russian Academy of Sciences, Chernogolovka MD, Russia ${ }^{3}$ Laboratoire de Physique des Solides, CNRS UMR 6502, Université de Paris-Sud, Orsay, France ${ }^{4}$ Departament de Química Física i Inorgànica, Universitat Rovira i Virgili, Marcel·li Domingo 1, 43007 Tarragona, Spain

5Institut de Ciència de Materials de Barcelona (ICMAB-CSIC), Campus de la UAB, 08193 Bellaterra, Spain

${ }^{6}$ Department of Chemistry and Biochemistry, University of California-Los Angeles, United
\end{abstract} States

email addresses of corresponding authors pawel.wzietek@u-psud.fr canadell@icmab.es, mgg@chem.ucla.edu, and patrick.batail@univ-angers.fr

\begin{abstract}
Here we present a study where what can be seen as a static modulation wave across arrays of crystalline 1,4-Bis( (4'(iodoethynyl)phenyl)ethynyl)

bicyclo[2,2,2]octane rotors changes the structure from one-half molecule to three-anda-half molecules in the asymmetric unit below a phase transition at $105 \mathrm{~K}$. The remarkable finding is that the total ${ }^{1} \mathrm{H}$ spin-lattice relaxation rate, $\mathrm{T}_{1}{ }^{-1}$, of unprecedented complexity to date in molecular rotors, is the weighted sum of the relaxation rates of the four contributing rotors

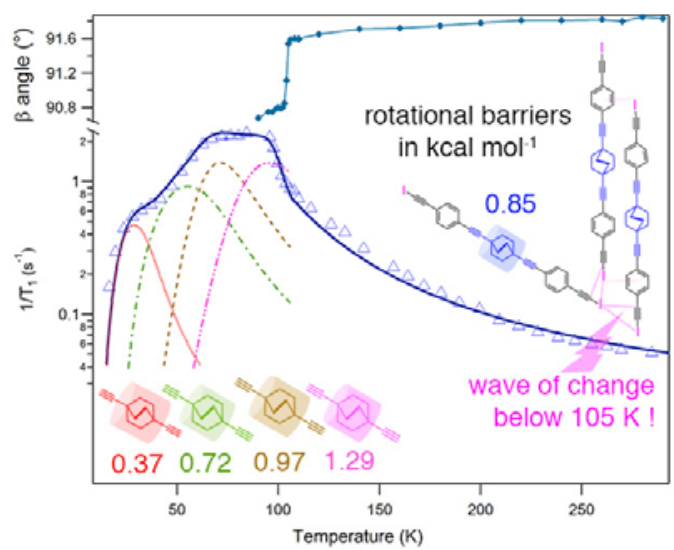
relaxation rates, each with distinguishable exchange frequencies reflecting Arrhenius parameters with different activation barriers (Ea) and attempt frequencies $\left(\tau_{0}^{-1}\right)$. This allows us to show, in tandem with rotorenvironment interaction energy calculations, how the dynamics of molecular rotors are able to decode structural information from their surroundings with remarkable nanoscale precision.
\end{abstract}




\section{Main Text}

The growth or turning movement of an organism, usually a plant, illustrates how highly organized structures tend to respond in a complex but reliable manner when subject to environmental stimuli. ${ }^{1}$ Understanding such events is important in the realms of materials chemistry and physics of molecular machines because the development of elaborate sensory devices with increasing levels of complexity is also required to accurately report changes that occur in the environment of their dynamic components. ${ }^{[2-6,8-10]}$

Here we present a study where what can be seen as a static modulation wave across parallel arrays ${ }^{[7,8]}$ of interacting iodine atoms in crystalline 1,4-Bis((4'(iodoethynyl)phenyl)ethynyl) bicyclo[2.2.2] octane rotors, $\mathbf{1}$ (Figure 1) changes the structure from one-half molecule to three-and-a-half molecules in the asymmetric unit below a phase transition at $105 \mathrm{~K}$. The remarkable finding is that the complex, total ${ }^{1} \mathrm{H}$ spinlattice relaxation rate, $T_{1}{ }^{-1}$, revealed by variable-temperature, variable-field experiments, is the weighted sum of the relaxation rates of the four contributing rotors relaxation rates. Hence, magnetic relaxation transforms from a process determined by a single molecular rotator in the high temperature phase into one that has distinguishable weighted contribution from the four new sites, each with distinguishable exchange frequencies reflecting Arrhenius parameters with different activation barriers (Ea) and attempt frequencies $\left(\tau_{0}{ }^{-1}\right)$. This allows us to show, in tandem with rotor-environment interaction energy calculations, how the dynamics of molecular rotors are able to decode structural information from their surroundings with remarkable nanoscale precision.
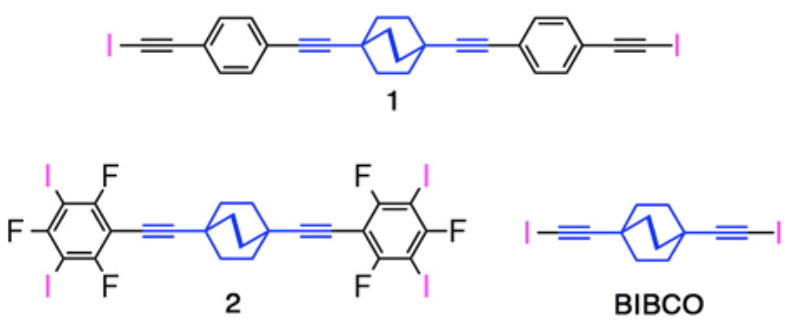

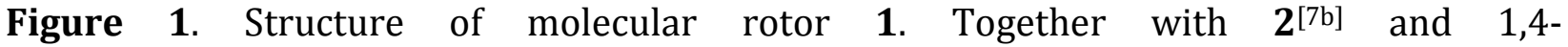
bis(iodoethynyl)bicyclo[2.2.2] octane (BIBCO) ${ }^{[8 \mathrm{a}]}$ they are a class of crystalline rotors that self-assemble by iodine...iodine interactions. Both $\mathbf{2}$ and BIBCO were previously shown to display ambient temperature dynamics in the GHz regime. Preparation and characterization of compound $\mathbf{1}$ are described in the Supporting Information section.

A set of three iodine...iodine interactions serves to direct the self-assembly of twodimensional arrays of $\mathbf{1}$ (Figure 2). While $\delta_{1}$ and $\delta_{2}$ are common to $\mathbf{1}$ and BIBCO (Figure S1), we show here that the installation of ethynylphenyl fragments on the BIBCO frame provides additional molecular degrees of freedom that, expressed through C-I $\cdots \pi$ interaction $\delta_{3},{ }^{[11]}$ confers a unique collective behavior to the dynamics of $\mathbf{1}$. 


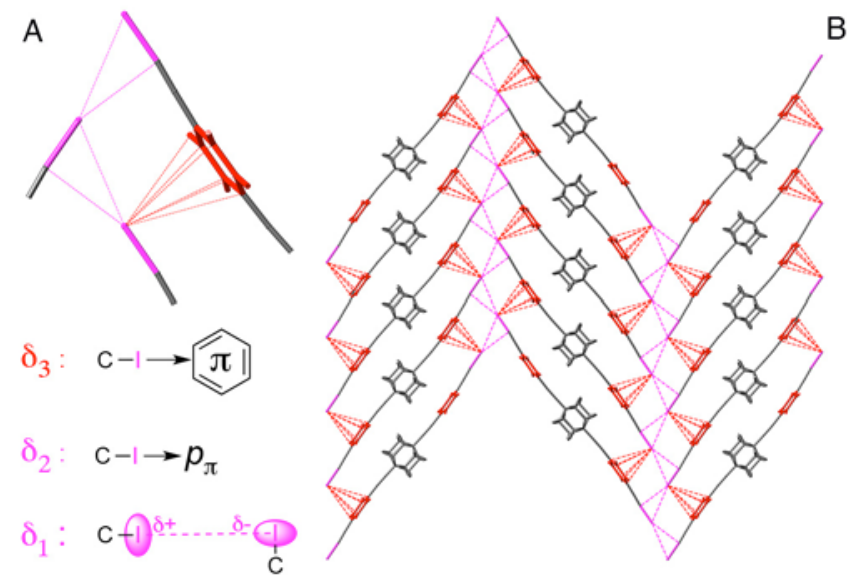

Figure 2. (A) The three types of iodine...iodine contacts and their schematic representations. (B) A single two-dimensional array self-assembled out of $\delta_{1}, \delta_{2}$ and $\delta_{3}$ in the structure of 1 at $120 \mathrm{~K}$. Note that only one out of the two equilibrium rotator positions are depicted here.

We have observed that the monoclinic unit cell of a single crystal of $\mathbf{1}$ abruptly changes, experiencing below $105 \mathrm{~K}$ (Figure $3 \mathrm{~B}$ ) an expansion by seven times its volume to encompass three and a half independent rotators at $90 \mathrm{~K}$, labeled I to IV in Figure 3C (only one-half independent rotor in the asymmetric unit is present in the structure of $\mathbf{1}$ at $120 \mathrm{~K}$; details of the investigation of the reversible transition and structure refinements at $120 \mathrm{~K}$, Figure 3A, and $90 \mathrm{~K}$, Figure 3C, can be found in the Supporting Information and Figures S4S5, S7-S8). It is even more remarkable that the layer-by-layer packing has each of the four homogenous, arrays of independent rotators stacked in alternating sequences, color-coded red (I), gold (II), blue (III) and black (IV) in Figure 3C. Moreover, the layers are stacked in registry yet alternately sliding by one-half molecular length. Thus, the inter-layer interactions primarily involve $\mathrm{C}-\mathrm{H} \cdots \mathrm{I}$ hydrogen bonds (Figure $3 \mathrm{~A}$ and $3 \mathrm{C}$ ) that define four distinct rotor-environment pockets. The origin of the differentiation of these pockets lies in what is seen as a static modulation wave of all three $\delta_{1}, \delta_{2}$ and $\delta_{3}$ halogen bonds encompassing the four homogenous layers of independent rotators I, II, III and IV, typically adjusting the degree of freedom of the $\delta_{3}$-bond manifold. For example, note that all phenyl rings are parallel at $120 \mathrm{~K}$; then see in Figure $\mathrm{S} 8$ the distribution of dihedral angles between two phenyl rings at $90 \mathrm{~K}$, from $0^{\circ}$ in IV, to $25.6^{\circ}, 14.7^{\circ}$ and $3.1^{\circ}$ in I, II and III, respectively. Moreover, the single C-I $\cdots \mathrm{I}-\mathrm{C}$ halogen bond, $\delta_{1}(\mathrm{I} \cdots \mathrm{I}, 4.049 \AA$ ) at $120 \mathrm{~K}$ bursts at the transition into seven different interactions whose I ...I distances (3.885 to $4.334 \AA$ ) may be seen more as halogen contacts ${ }^{7 f}$ yet are similar to those that direct the self-assembly and halogen-bond mediated phase diagrams of $2^{8 b}$ and BIBCO. ${ }^{8 a}$ 


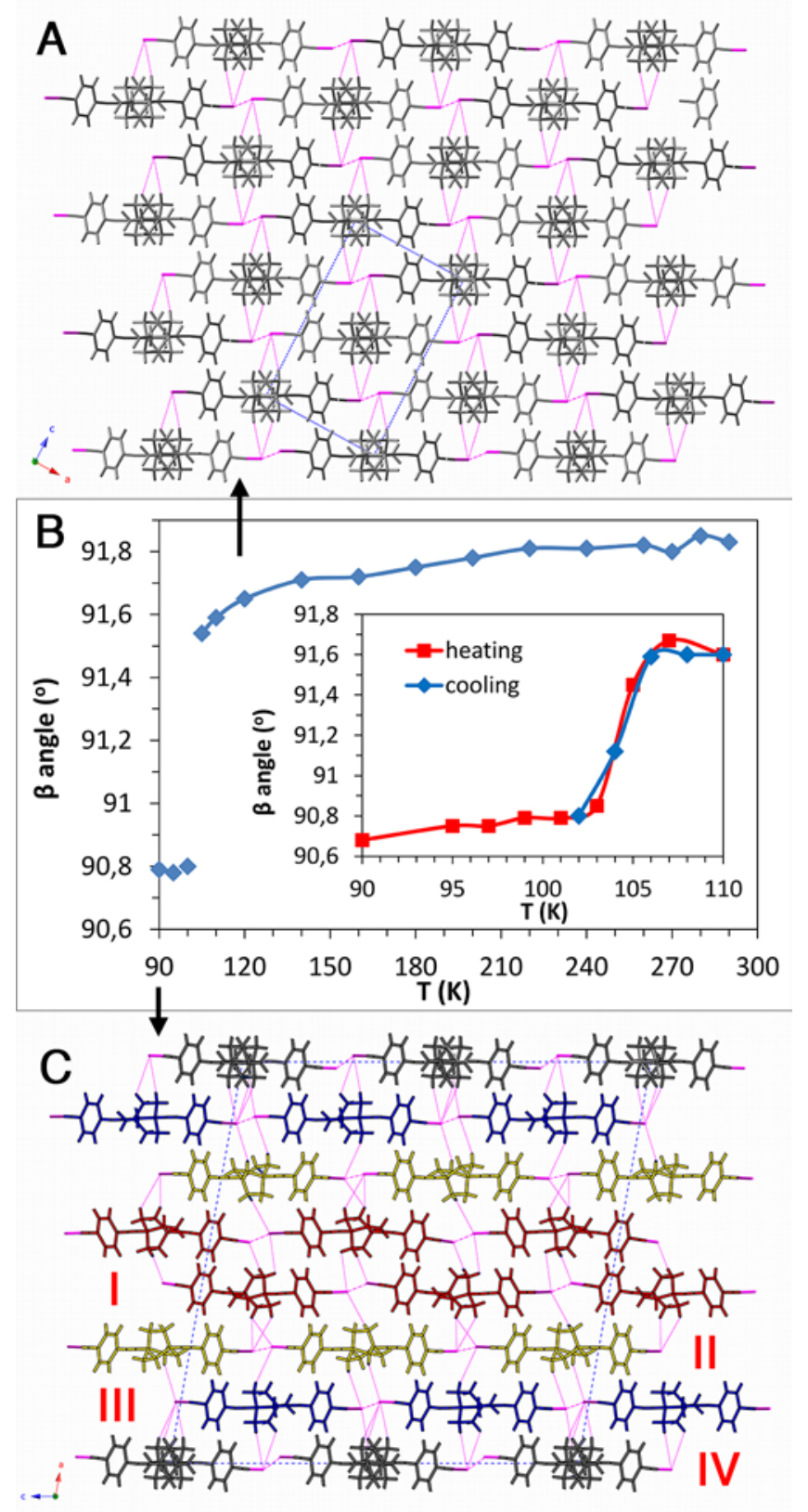

Figure 3. (A) Layer-by-layer packing of arrays of the single one-half independent rotators (disordered with balanced occupancies on two equilibrium positions) in the structure of 1 at $120 \mathrm{~K}$. Dotted red lines are $\delta_{1}$ interactions and $\mathrm{C}-\mathrm{H} \cdots \mathrm{I}$ hydrogen bonds by which the rotators rub on their environment. (B) The reversible structure transition (see also Figures S4-S6) illustrated by an abrupt change of the monoclinic angle $\beta$. (C) Below the transition, a static modulation wave of the three halogen-bonding interactions encompasses a registry of four different arrays (I to IV) shown here at $90 \mathrm{~K}$. Note that only one rotator (IV) is disordered with balanced occupancies while all three other independent rotators have one equilibrium position only. 
Variable-field ${ }^{1} \mathrm{H}$ and ${ }^{13} \mathrm{C}$ solid state NMR experiments carried out on static crystalline samples over large temperature and pressure windows have illuminated our understanding of the balance of competing electronic instabilities coupled to molecular degrees of freedom at the heart of the rich quantum physics in low dimension in strongly correlated molecular conductors. ${ }^{[12]}$ Translating to the realm of molecular rotors the ability to perform variable-field, variable-temperature proton spin-lattice relaxation time experiments, ${ }^{1} \mathrm{H} T_{1}{ }^{-1}$, has recently demonstrated great promise to gain insight into the dynamics of crystalline rotors and help disentangle multiple relaxation processes eventually occurring all at once at the same spin temperature (see the basic principles in the Supporting Information). ${ }^{88,9,10]}$ Here, variable-temperature $(20-300 \mathrm{~K})$ proton spinlattice relaxation experiments carried out at two ${ }^{1} \mathrm{H}$ Larmor frequencies on a polycrystalline sample of 1 reveal at the temperature of the structural transition a reversible discontinuity (Figure 4) similar to that occurring in $2,{ }^{[8 b]}$ another extended analog of BIBCO also endowed with the additional degree of freedom conferred by two phenyl rings implemented in the rod axle (Figure 1).
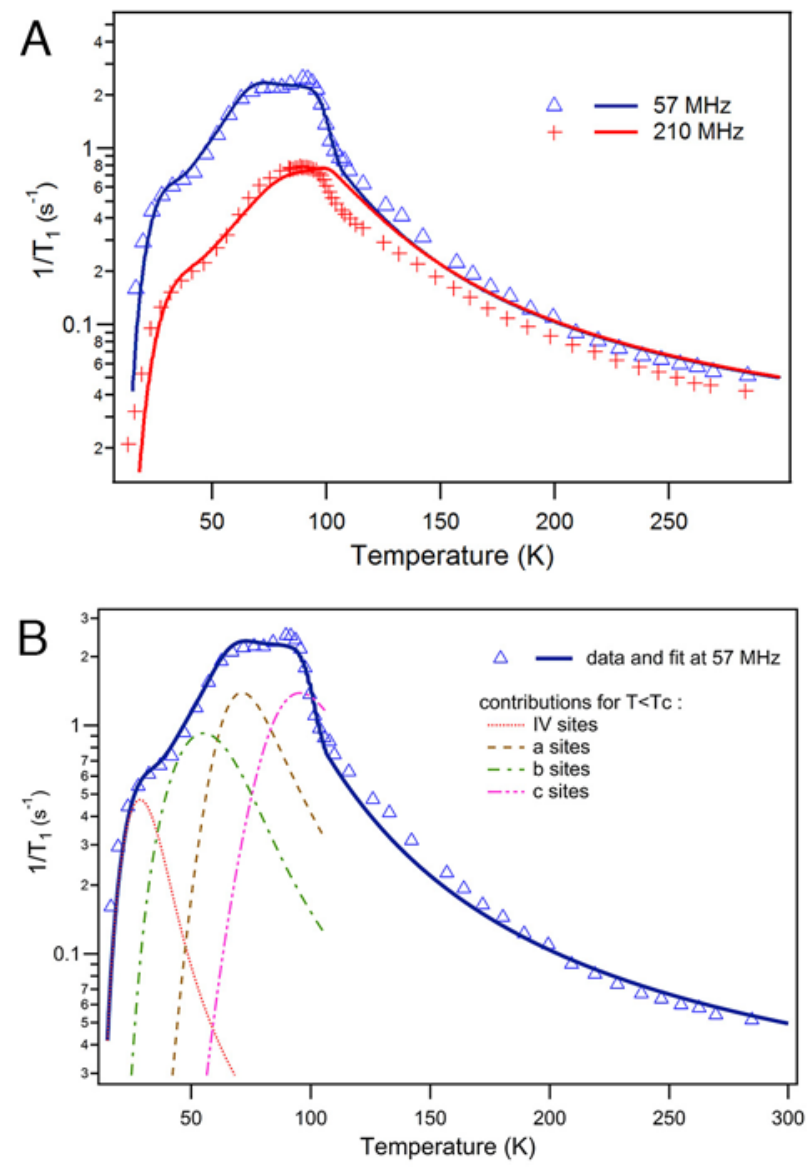

Figure 4. (A) Variable-temperature ${ }^{1} \mathrm{H}$ spin-lattice relaxation time $\mathrm{T}_{1}{ }^{-1}$ at 57 and $210 \mathrm{MHz}$ for 1. The blue and red lines represent the fits ${ }^{[13]}$ of the data to a sum of standard KuboTomita expressions, with thermally activated correlation times $\tau_{\mathrm{i}}=\tau_{0} \exp \left(E_{i} / \mathrm{kT}\right)$ where $E_{i}$ 
denotes the energy barrier at site $i$ (see text and Supporting Information). (B) Curves for each of the four components of the total relaxation $\left(T<T_{\mathrm{c}}\right)$ drawn for one frequency.

Below the phase transition, the $\mathrm{T}_{1}{ }^{-1}$ data display several entangled maxima; this is not unexpected since the analysis of the structure at $90 \mathrm{~K}$ led us to explicit an inventory of a total of four independent rotator sites that would result in complex dynamics encompassing as many independent relaxation processes. The intensity of $\mathrm{T}_{1}^{-1}$ maxima scales with the net number of moving ${ }^{1} \mathrm{H}$ on each independent site; hence, the fits of the data to the KuboTomita (KT) formula for $\mathrm{T}_{1}$ with correlation times $\tau_{\mathrm{i}}=\tau_{0} \exp \left(E_{\mathrm{i}} / \mathrm{k} T\right)$ were conducted with the knowledge that, below the transition, rotators I, II and III each involves 2/7 of the total number of moving protons, rotators IV having $1 / 7$ of the net number. Therefore, the low temperature data can be fitted with a weighted sum of four KT expressions with different energy barriers (Figure $4 \mathrm{~B}$ and Supporting Information), hereafter noted $E_{\mathrm{a}}, E_{\mathrm{b}}, E_{\mathrm{c}}, E_{\mathrm{IV}}$. Note that the first three barriers refer to rotators I, II and III and cannot be assigned solely from the fits because they have the same weight. On the other hand, all moving protons are located on a single independent site at $120 \mathrm{~K}$, therefore above the transition temperature, $T \mathrm{c}$, the fit was done assuming a single barrier, noted $E_{\mathrm{HT}}$. To simplify the fits, all processes were assigned a common attempt frequency $\left(\tau_{0}{ }^{-1}\right)$; this is expected for identical rotors since $\tau_{0}$ is a frequency characteristic of a specific, freely moving rotor. The best fit shown in Figure 4A was obtained with $\tau_{0}=1.8 \times 10^{-12} \mathrm{~s}$.

For $T>T_{\mathrm{c}}$, the fit yields $E_{\mathrm{HT}}=429 \mathrm{~K}\left(0.85 \mathrm{kcal} \mathrm{mol}^{-1}\right)$. For the low temperature phase, we could not obtain good fits with fixed barriers, which let us to assume the existence of a slight disorder implying a distribution of energy barriers. A Gaussian distribution was then set for each barrier, with the $\sigma$ values of their respective relative widths given below in percent of the barrier values. Likewise, we assumed a slight distribution of the transition temperature $T_{\mathrm{c}}$ in the fit. The fits shown in Figure 4 yield $E_{\mathrm{IV}}=188 \mathrm{~K}\left(0.37 \mathrm{kcal} \mathrm{mol}^{-1}\right), \sigma_{\mathrm{IV}}=$ $20 \%$; and three additional energy barriers $E_{\mathrm{a}}=360 \mathrm{~K}\left(0.72 \mathrm{kcal} \mathrm{mol}^{-1}\right), \sigma_{\mathrm{a}}=22 \% ; E_{\mathrm{b}}=486 \mathrm{~K}$ $\left(0.97 \mathrm{kcal} \mathrm{mol}^{-1}\right), \sigma_{\mathrm{b}}=1.2 \% ; E_{\mathrm{c}}=651 \mathrm{~K}\left(1.29 \mathrm{kcal} \mathrm{mol}^{-1}\right), \sigma_{\mathrm{c}}=1.9 \% ; \mathrm{Tc}=100 \mathrm{~K}, \sigma_{T \mathrm{c}}=3.6 \%$ (Table S2) to be assigned to the motion of rotators in sites within layers I, II and III.

Assigning relaxation processes at the nano- and micro-scale, be they discrete events on distinct sites $[8 \mathrm{a}, \mathrm{b}, 9 \mathrm{a}, \mathrm{c}]$ or dual, coupled processes associated with correlated rotational motion in well-separated pairs of rotators, ${ }^{[9 b, 10]}$ requires the fits to the $\mathrm{T}_{1}{ }^{-1}$ data to be coupled to calculations ${ }^{[8 \mathrm{~b}, 9-10]}$ of rotor-environment interaction energies (Table S2). Thus, we have estimated the five rotational barriers (Figure 5) for each independent rotator by means of partial geometry optimizations (rotor itself and the nearest molecules of the environment interacting with it through I atoms) using the same computational details reported previously.[9a] 


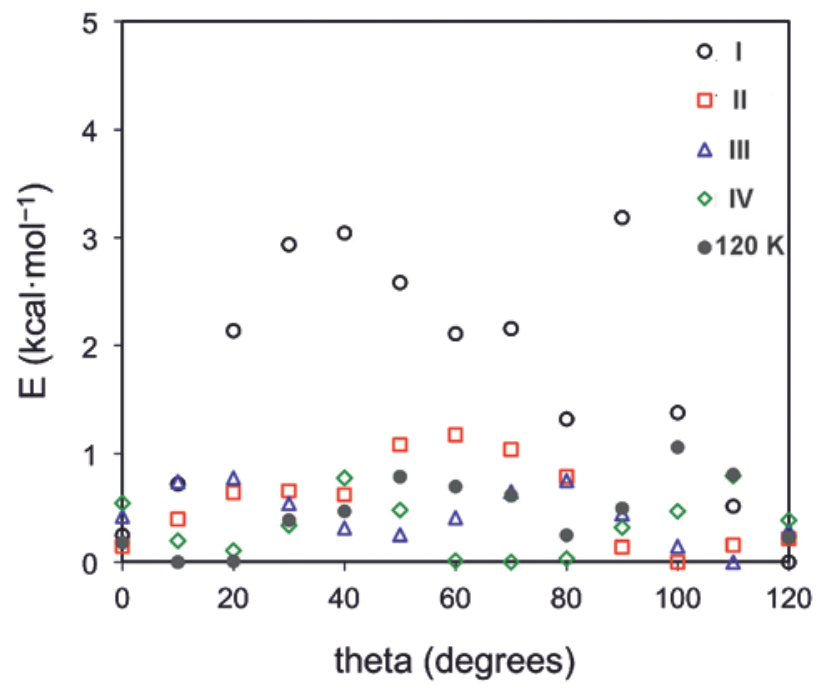

Figure 5. Computed energy profiles (in $\mathrm{kcal} \mathrm{mol}^{-1}$ ) for the rotation of each independent rotator surrounded by their neighbor molecules; the five models for the DFT transition structures are described in Figure S11.

All barriers (Figure 5 and Figure S11) are calculated around $1 \mathrm{kcal} \mathrm{mol}^{-1}(0.78-1.18)$ except that for rotator I in the $90 \mathrm{~K}$ structure which is noticeably higher at $3.19 \mathrm{kcal} \mathrm{mol}^{-1}$ (1605 K). This is because shorter $\mathrm{H} \cdots \mathrm{I}$ contacts occur in the transition state for I, including a contact as short as $2.94 \AA$. In contrast, rotators in sites II, III and IV have no contact shorter than $3.2 \AA$ when the geometry of their transition structures is optimized along their rotational trajectories. For example, the shorter $\mathrm{H} \cdots \mathrm{I}$ contact for II, the $120 \mathrm{~K}$ rotator, and IV are 3.20-3.21 Å; they are even larger, $3.28 \AA$ for III. Therefore, the activation barriers seem to be primarily determined by the strength of the shorter $\mathrm{H} \cdot \cdots \mathrm{I}$ contact in their transition state.

Moreover, the values of the calculated barriers in Figure 5 allow for the assignment (Table S2 and Figure S11) of the rotational barriers extracted from the fits of the $T_{1}{ }^{-1}$ data between $20 \mathrm{~K}$ and $300 \mathrm{~K}$; both are fully coherent and qualify a global rotor dynamics characterized by one large rotational barrier supplemented by two pairs of smaller ones separated by $\sim 124 \mathrm{~K}$ (proton spin-lattice experiments) or $\sim 166 \mathrm{~K}$ (rotor-environment energy calculations). The remarkable agreement demonstrated here testifies the validity of this integrated approach.

Beyond the nanoscale change stimuli of the present crystalline rotor dynamics, providing information on their surroundings with a remarkable level of accuracy, other concerted changes are hidden in rich phase diagrams to be disclosed by opening large temperature windows, integrating structure, spin-lattice relaxation, dielectric and secondorder nonlinear optical experiments, and rotor-environment energy calculations. Unraveling the phase diagrams of these materials, also tackling in future work their response to uniaxial and isostatic pressure, is interesting because they could be used as simulators to control molecular machines.

In addition to drawing attention to the susceptibility of extended, non-covalent halogen-bond arrays to undergo concerted modulation in their temperature phase 
diagrams, $[8 \mathrm{a}, \mathrm{b}]$ the results observed with compound $\mathbf{1}$ provide[8a,b,9,10] the kind of in-depth insight on the mechanism of motion required on the way towards a control of molecular machines at the nanoscale.[2-6]

\section{Supporting Information}

Supporting Figures and Tables, Synthesis, Crystal structures at $120 \mathrm{~K}$ and $90 \mathrm{~K}$, VT ${ }^{1} \mathrm{H}$ spinlattice relaxation time: basic principles and experimental details, computational details. CCDC 1577087 and 1577088 contain the crystallographic data for this paper. These data can be obtained free of charge from The Cambridge Crystallographic Data Centre.

\section{Acknowledgments}

This research was funded by the CNRS, the University of Angers, the University of Paris-Sud, Orsay; the Région des Pays de la Loire Grant MOVAMOL, the joint CNRS-Russian Federation grants PICS 6028 and RFBR-CNRS 12-03-91059 (Chernogolovka). Work at UCLA was supported by USA National Science Foundation grants DMR140268 and DMR-1700471. Work in Bellaterra and Tarragona was supported by the Spanish Ministerio de Economía y Competitividad (Grants FIS2015-64886-C5-4-P and CTQ2014-52774-P) and Generalitat de Catalunya (2017SGR1506 and 2017SGR629). E.C. acknowledges support by MINECO (Spain) through the Severo Ochoa Centers of Excellence Program (Grant SEV-2015-0496).

S.S.; C. L. and G. B.; thank the CNRS and the Région des Pays de la Loire for a post-doctoral and Ph. D. grants, respectively. L. Z. thanks the CNRS for an Associated Researcher Fellowship.

\section{Conflict of interest}

The authors declare no conflict of interest.

\section{References}

[1] (a) Kocsis, L. S.; Elbel, K. M.; Hardigree, M. A.; Brummond, K. M.; Haidekker, M. A.; Theodorakis,E. A. Org. Biomol. Chem. 2015, 13, 2965; and reference 1 therein. (b) Pickard, B. G. Current Topics in Membranes 2007, 58, 361.

[2] Abendroth, J. A.; Bushuyev, O. S.; Weiss, P. S.; Barrett, C. J. ACS Nano 2015, 9, 7746.

[3] Erbas-Cakmak, S.; Leigh, D. A. ; McTernan, C. T. ; Nussbaumer, A. L. Chem. Rev. 2015, 115, 10081.

[4] Domingos, S. R.; Cnossen, A.; Buma, W. J.; Browne, W. R.; Feringa, B. L.; Schnell, M. Angew. Chem. Int. Ed. 2017, 56, 11209.

[5] Nishiyama, H.; Takeda, T.; Hoshino, N.; Takahashi, K.; Noro, S.-I.; Nakamura, T.; Akutagawa, T. Cryst. Growth Des. 2018, 18, 286.

[6] Vogelsberg, C. S.; Garcia-Garibay, M. A. Chem. Soc. Rev. 2012, 41, 1892.

[7] Selected references on halogen bonding: (a) Legon, A. C. Phys. Chem. Chem. Phys. 2010, 12, 7736. (b) Fourmigué, M. Curr. Opinion Sol. St. Mater. Sc. 2009, 13, 36. (c) Bertani, R.; Sgarbossa, P.; Venzo, A.; Lelj, F.; Amati, M.; Resnati, G.; Pilati, T.; Metrangolo, P.; Terraneo, G. Coord. Chem. Rev. 2012, 254, 677. (d) Troff, R. W. ; Mäkelä, T.; Topic, F.; Valkonen, A.; Raatikainen, K.; Rissanen, K. Eur. J. Org. Chem. 2013, 1617. (e) Meyer, F.; Dubois, P. CrystEngComm 2013, 15, 3058. (f) Desiraju, G. R.; Shing Ho, P.; Kloo, L.; Legon, A. C.; Marquardi, R.; Metraangelo, P.; Politzer, P.; Resnati, G.; Rissanen, K. Pure Appl. Chem. 2013, 85, 1711. 
[8] Halogen interactions in crystalline rotors: (a) Lemouchi, C.; Vogelsberg, C.; Simonov, S.; Zorina, L.; Batail, P.; Brown, S.; Garcia-Garibay, M. J. Am. Chem. Soc. 2011, 133, 6371. (b) Lemouchi, C.; Yamamoto, H. M.; Kato, R.; Simonov, S.; Zorina, L.; RodríguezFortea, A.; Canadell, E.; Wzietek, P.; Iliopoulos, I.; Gindre, D.; Chrysos, M.; Batail, P. Cryst. Growth Des. 2014, 14, 3375. (c) Catalano, L.; Peŕez-Estrada, S.; Terraneo, G.; Pilati, T.; Resnati, G.; Metrangolo, P.; Garcia-Garibay, M. A. J. Am. Chem. Soc. 2015, 137, 18386. (d) Catalano, L.; Perez-Estrada, S.; Wang, H.-H.; Ayitou, A. J.-L.; Khan, S. I.; Terraneo, G.; Metrangolo, P.; Brown, S.; Garcia-Garibay, M. A. J. Am. Chem. Soc. 2017, 139, 843.

[9] (a) Lemouchi, C.; Mézière, C.; Zorina, L.; Simonov, S.; Rodríguez-Fortea, A.; Canadell, E.; Wzietek, P.; Auban-Senzier, P.; Pasquier, C.; Giamarchi, T.; Garcia-Garibay, M. A.; Batail, P. J. Am. Chem. Soc. 2012, 134, 7880. (b) Lemouchi, C.; Iliopoulos, K.; Zorina, L.; Simonov, S.; Wzietek, P.; Cauchy, T.; Rodríguez-Fortea, A.; Canadell, E.; Kaleta, J.; Michl, J.; Gindre, D.; Chrysos, M.; Batail, P. J. Am. Chem. Soc. 2013, 135, 9366. (c) Bastien, G.; Lemouchi, C.; Wzietek, P.; Simonov, S.; Zorina, L.; Rodríguez-Fortea, A.; Canadell, E.; Batail, P. Zeit. Anorg. Allg. Chem. 2014, 640, 1127-1133.

[10] (a) Bastien, G.; Lemouchi, C.; Allain, M.; Wzietek, P.; Rodríguez-Fortea, A.; Canadell, E.; Iliopoulos, K.; Gindre, D.; Chrysos, M.; Batail, P. CrystEngComm 2014, 16, 1241. (b) Kaleta, J.; Michl, J.; Mézière, C.; Simonov, S.; Zorina, L.; Wzietek, P.; Rodríguez-Fortea, A.; Canadell, E.; Batail, P. CrystEngComm 2015, 17, 7829. (c) Rodriguez-Fortea, A.; Kaleta J.; Mézière, C.; Allain, M.; Canadell, E.; Wzietek, P.; Michl, J.; Batail, P. ACS Omega 2018, 3, 1293-1297.

[11] Cao, J.; Yan, X.; He, W.; Li, X.; Mo, Y.; Liu, M.; Jiang, T.-B. J. Am. Chem. Soc. 2017, 139, 6605.

[12] See, for example: (a) Zorina, L.; Simonov, S.; Mézière, C.; Canadell, E.; Suh, S.; Brown, S. E.; Foury-Leylekian, P.; Fertey, P.; Pouget, J.-P.; Batail, P. J. Mater. Chem. 2009, 19, 6980-6994. (b) Baudron, S. A.; Batail, P.; Coulon, C.; Clérac, R.; Canadell, E.; Laukhin, V.; Melzi,R.; Wzietek, P.; Jérome, D.; Auban-Senzier, P.; Ravy, S. J. Am. Chem. Soc. 2005, 127, 11785-11797. (c) Miyagawa, K.; Kanoda, K.; Kawamoto, A. Chem. Rev. 2004, 104, 5635-5653. (d) Lefebvre, S.; Wzietek, P.; Brown, S.; Bourbonnais, C.; Jérome, D.; Mézière, C.; Fourmigué, M.; Batail, P. Phys. Rev. Lett. 2000, 85, 5420-5423. (e) Mayaffre, H.; Wzietek, P.; Lenoir, C.; Jérome, D.; Batail, P. Europhys. Lett. 1994, 28, 205-210.

[13] Multi-parameter fits of the NMR relaxation were done using a genetic optimisation algorithm; see for example: (a) Wormington, M.; Panaccione, C.; Matney, K. M.; Bowen, D. K. Phil. Trans. R. Soc. Lond. A 1999, 357, 2827-2848. (b) Nelson, A. J. Appl. Cryst. 2006, 39, 273. 